\title{
Note on the generalized form of Weber's Law
}

JOHN A. MICHON 1

INSTITUTE FOR PERCEPTION, SOESTERBERG, NETHERLANDS

In this note it is argued that $\Delta I=k\left(I+I_{n}\right)$ as an expression for the generalized Law of Weber (Treisman, 1964) is confusing. The expression $\Delta I=k I P+i_{n}$ should be preferred in the light of the evidence available.

In several papers, such as Barlow (1957) and Treisman (1964), we find a somewhat puzzling formulation for the "generalized" Law of Weber:

$$
\Delta I=k\left(I+I_{n}\right) p
$$

in which $I_{n}$ represents the spontaneous activity of the visual system in absence of external stimulation, and $p(0.5 \leq p \leq 1)$ depends on the range of the stimuli (at least for brightness discrimination). The following arguments will show that formulation (1) is confusing and that Weber's Law in its general form should preferably read:

$$
\Delta I=\left.k\right|^{P}+i_{n}
$$

in which $i_{n}$ is the threshold residue which is not affected by $k$ or $p$ in this case. This threshold may, incidentally, be thought of as $i_{n}=c . i_{o}$, where $c$ is a constant $\doteq 1$, depending on the experimental method used (Miller, 1947).

(a) It has been found that brightness discrimination data fit a square root relation when the stimuli are of very low intensity. The original conception of De Vries and Rose was to consider the eye as a perfect physical detector, in which case $\Delta I=\mathrm{kI}^{0.5}$, as a consequence of the statistical fluctuations in the incidence of light quanta on the retina. A threshold noise $i_{n}$ may then simply be added, to account for the spontaneous activity of the visual system (Treisman, 1964; Bouman, Vos, \& Walraven, 1963; Latour, 1966). In this conception, the square root relation has an external cause, and the activity of the visual system reflects this, adding its own, random, activity. If we adopt expression (1) however, the origin of the square root relation becomes ambiguous. Instead of the statistical fluctuations in the stimulus itself, a different mechanism must be hypothesized to generate the square root relation. This mechanism must be of a more central nature than the processes causing the "Augengrau" or "dark light," in order to account for the factor $I_{n}{ }^{0.5}$ in equation (1). This would destroy the elegance of the original model of De Vries and Rose. Moreover it is not explicitly proposed by Barlow (1957) as an alternative to that model.

(b) Instead of equation (2) we may use the expression $\Delta I=k\left(I^{p}+I_{n}\right)$, in which the constant $I_{n}$ is expressed in terms of the stimulus intensity. In stimulus dimensions other than light where such a residual stimulation is not easily identified, such as weight lifting or time estimation, it seems that expression (2) is preferable, since it leaves open from what source the noise originates. It may rather be considered as expressing uncertainty in the psychophysical judgments made by the subject. Failing to take the formal distinction between the expressions into account will result in confusion. An example from another study by Treisman (1963) will illustrate this. From his experiments on time perception Treisman concludes that Weber's Law holds for short time intervals. For two different conditions the relations derived are $\Delta \mathrm{T}=0.057(\mathrm{~T}+$ $0.900)$ and $\Delta T=0.075(T+0.501)$. With reference to Stroud's "psychological moment" hypothesis-which may be considered as a statement about the absolute threshold for duration-Treisman (1963, p. 28) concludes that "the values of $a$ [i.e., $I_{n}$ in the present text-JAM ] obtained experimentally are considerably larger than the perceptual moment, which is estimated to be 50-200 msec. long." If we adopt (2) then, instead of estimates of 0.900 and 0.501 sec. for $a\left[=I_{n}\right]$, we obtain 52 and $38 \mathrm{msec}$. respectively for $a^{\prime}\left[=i_{n}\right]$ sufficiently close to Stroud's quite arbitrary limits.

(c) A testable consequence of expression (1) is that it predicts an inhibiting effect of I on the spontaneous activity of the visual system: when I increases, the relative weight of $I_{n}$ on the value of $\Delta I$ will decrease quadratically instead of linearly as predicted by (2). If we compare the behavior of the functions (1) and (2) in the range where $I \doteq I_{n}$ or $I \doteq i_{n} / k$, we see that the transition from the asymptote $\Delta \mathrm{I}=\mathrm{C}$ to the asymptote $\Delta \mathrm{I}=\mathrm{kJ}^{0.5}$ is much sharper according to (1) than it is when (2) holds. Though no experimental data in this range of stimulation are available at present, the consequences of the assumptions are in principle open to verification.

In summary we reach the conclusion that in absence of experimental evidence favoring expression (1), expression (2) should be preferred as a general expression for Weber's Law. Meanwhile the points raised in this note by themselves should not invalidate the conclusions drawn by Treisman (1964) with respect to the merhanisms of sensory discrimination.

\section{References}

Barlow, H. B. Increment thresholds at low intensities considered as signal/noise discrimination. J. Physiol., 1957, 136, 469-488. Bouman, M. A., Vos, J. J., \& Walraven, P. L. Fluctuation theory of luminance and chromaticity discrimination. J. Opt. Soc. Amer., $1963,53,121-128$.

Latour, P. L. A comparison of two visual threshold models. In Studies in perception dedicated to $M$. A. Bouman, Soesterberg: Institute for Perception RVO-TNO, 1966. Pp. 93-104. 
Miller, G. A. Sensitivity to changes in the intensity of white noise and its relation to masking and loudness. J. Acoust. Soc. Amer. $1947,19,609-619$.

Treisman, M. Temporal discrimination and the indifference interval: Implications for a model of the "internal clock." Psychol. Monogr., 1963, 77, No. 13 (Whole No. 576).

Treisman, $M$. Noise and Weber's Law: The discrimination of brightness and other dimensions. Psychol. Rev., 1964, 71, 314-330.

\section{Note}

1. This note was prepared while the author was at Carnegie Institute of Technology, Pittsburgh, Pennsylvania as recipient of a NATO Science Fellowship, granted by the Netherlands Organization for Pure Scientific Research.

(Received for publication, August 4, 1966.) 